\title{
Effet des faibles doses des rayonnements ionisants sur l'homme
}

\author{
Effect of low dose ionising radiation on humans
}

\author{
Bernard Le Guen \\ Électricité de France, EDF, DPN, Cap Ampère, 1 place Pleyel, 93282 Saint Denis Cedex, France
}

Résumé - Les textes réglementaires actuels et futurs sur les expositions aux rayonnements ionisants à faibles doses et débits de dose font l'hypothèse implicite de la persistance d'un risque résiduel quel que soit le niveau d'exposition. Ils sont basés sur les études épidémiologiques des survivants de Hiroshima et de Nagasaki, études compatibles avec une relation linéaire entre l'exposition aux radiations et l'excès de tumeurs à partir d'une exposition correspondant à environ 200 mSv. La difficulté est de savoir si la linéarité reste l'hypothèse la plus vraisemblable pour l'évaluation du risque pour des doses cumulées et des débits de dose inférieurs à 200 mSv.

Le développement de la biologie moléculaire a ouvert de nouvelles perspectives permettant de mieux connaître les mécanismes biologiques à l'origine des cancers, pour exemple, la connaissance des conséquences de l'acquisition de l'instabilité génomique sur la cellule. Les hypothèses actuelles de cancérisation s'expliquent plus par un « effet de champ » et de communication inter cellulaire (effet by-stander) que par l'irradiation d'un seul gène ou d'une seule cellule. Les variations de radiosensibilité individuelle proviennent probablement de la variabilité interindividuelle à réparer les lésions de l'ADN ou à éliminer les cellules endommagées. Cependant, la découverte des gènes suppresseurs de tumeurs et de leur fonction a amené à identifier les sujets plus prédisposés aux cancers car porteurs constitutionnellement d'un allèle muté de l'un de ces gènes.

Les études épidémiologiques permettent d'estimer directement le risque de cancer chez des travailleurs exposés à de faibles doses de rayonnement mais seront toujours limitées pour estimer l'effet des faibles doses du fait notamment des facteurs confondants, par conséquent la compréhension des mécanismes cellulaires de cancérogenèse reste fondamentale pour l'estimation du risque biologique.

Mots clés - Faible dose, rayonnement ionisant, études biologiques, effet bystander, instabilité génomique, risque de cancer

\footnotetext{
Abstract - Present regulations on exposure to low level and low rate ionising radiation are based on a hypothetical residual risk even at low doses. The regulations are based on epidemiological studies on Hiroshima and Nagasaki survivors. These studies are compatible with a Linear No Threshold (LNT) dose-effect relation for exposure of around 200 mSv.

The difficulty is to determine whether this hypothesis is true for the risk evaluation for cumulated doses and doses rate below 200 mSv.

The advances in molecular biology have opened new perspectives for understanding biological mechanisms at the origin of cancer such as the knowledge of genomic instability
} 
on cells. The current carcinogenesis hypothesis after exposure are based on the tissue answer and bystander effect rather than irradiation of a single gene or cell.

Variation in individual radio sensitivity is probably due to the variation in capacity to repair DNA damage or to eliminate damaged cells.

However, the discovery of tumour suppressor genes and their function has allowed to identify subjects predisposed to cancer due to mutated genes in stem cells.

Epidemiological studies can estimate cancer risk in workers exposed to low dose radiation. However confounding factors limit the understanding of the effect of low doses on health. This is why it's important to continue the biological research in order to better assess low doses risk.

Key words - low doses, ionising radiation, biological studies, genomic instability, bystander effect, cancer risk

\section{INTRODUCTION}

Quel que soit le lieu et le mode de vie, nul ne peut échapper tout au long de son existence à une radioactivité ambiante variable mais constante. Elle participe à notre environnement depuis sa création. L'organisme entretient en permanence une radioactivité interne naturelle. Son origine peut être cosmique ou terrestre, témoignage de la création de l'Univers et de la persistance des radioéléments à très longue période, essentiellement le potassium 40 et les chaînes radioactives de l'uranium et du thorium.

Les effets nocifs sur la santé de la radioactivité et plus généralement de toute irradiation, même à faible dose, constituent généralement la principale crainte suscitée par l'énergie nucléaire. Dans cet article, sont présentés succinctement les fondements scientifiques sur lesquels reposent les textes réglementaires et sont exposées les connaissances actuelles sur des processus impliqués dans les effets biologiques des rayonnements ionisants.

\section{UNE RÉGLEMENTATION BASÉE SUR L'ÉVOLUTION DES CONNAISSANCES}

Les textes réglementaires actuels et futurs sur les expositions aux rayonnements ionisants à faibles doses et débits de dose font l'hypothèse implicite de la persistance d'un risque résiduel quel que soit le niveau d'exposition. Ils sont basés sur les études épidémiologiques des survivants de Hiroshima et de Nagasaki.

Pour succéder en 1975 à l'ABCC (Atomic Bomb Casualty Commission), les américains et les japonais ont créé la RERF (Radiation Effects Research Foundation). Les travaux de cette fondation ont permis le suivi à long terme des survivants aux deux explosions nucléaires de Hiroshima et Nagasaki, à l'origine de l'évolution de la réglementation.

Une dosimétrie individuelle (DS 86) a pu être reconstituée pour 86572 habitants. Cette cohorte est essentiellement composée d'une population faiblement exposée, inférieure à 0,3 Gy délivrée instantanément (Fig. 1). Des incertitudes concernent l'estimation de l'exposition aux neutrons. 


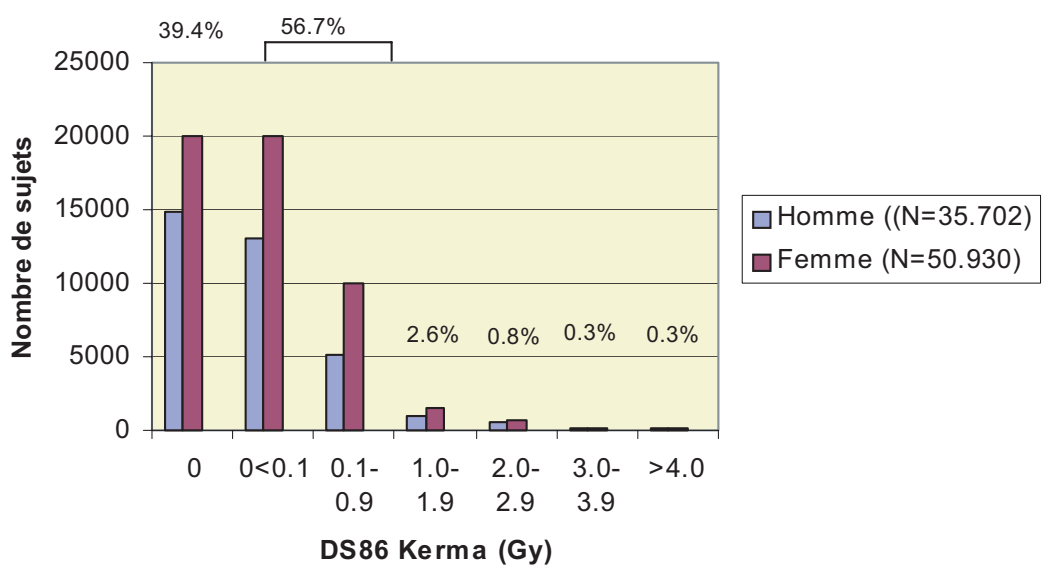

Fig. 1. Distribution des sujets selon l'estimation de dose DS86 (Hiroshima + Nagasaki).

Fig. 1. Distribution of number of subjects according to dose DS86 (Hiroshima + Nagasaki).

Ces programmes de suivi réalisés sur une large échelle, 120000 personnes au total dont 80000 enfants et 3300 exposés in-utéro au moment de l'explosion, ont pris en compte la morbidité et la mortalité des survivants de 1950 à nos jours (LSS : Life Span Study). Rappelons pour mémoire qu'en 1994, 49 \% étaient encore en vie.

L'excès de cancers est très faible (335 tumeurs solides et 83 leucémies : Tab. 1) par rapport aux conséquences immédiates des explosions qui ont dévasté les deux cités japonaises (210000 morts pour 610000 habitants...).

II n'y a pas un cancer spécifique dû aux radiations ionisantes mais des cancers des organes digestifs (estomac et colon), du poumon, du sein et des voies urinaires qui apportent l'essentiel de la contribution à l'excès global.
Le développement au cours des dernières années de la biologie moléculaire ouvre de nouvelles perspectives permettant de mieux connaître les mécanismes biologiques à l'origine des cancers. La RERF se lance dans ce nouveau champs d'action. Les études génétiques portant sur la banque d'ADN de plus de 1000 familles particulièrement suivies, permettront la compréhension des effets à long terme sur la descendance.

RERF. L'étude sur l'incidence des cancers a bénéficié d'un suivi jusqu'en 1995 (soit 8 ans supplémentaires) et les études de mortalité ont été prolongés de 7 ans (1990-1997) (Preston, 2003).

Les conclusions sont que les hypothèses d'un excès de risque sur la vie entière pour les cancers solides ne sont pas fondés mais que l'excès de risque par Sv, relatif ou absolu, décroît avec l'age atteint, de l'ordre de $20 \%$ par tranche de 10 ans. 
Tableau 1. Tumeurs solides et leucémies en fonction de la dose 1950-1990.

Table 1. Solid tumors and leukaemia according to dose (1950-1990).

\begin{tabular}{|c|c|c|c|c|c|c|c|c|}
\hline & \multicolumn{4}{|c|}{ Tumeurs solides } & \multicolumn{4}{c|}{ Leucémies } \\
\hline Dose (Sv) & Sujets & Observées. & Attendues & Excès & Sujets & Observées. & Attendues & Excès \\
\hline$<0,005$ & 36459 & 3013 & 3054 & -41 & 35458 & 73 & 65 & 8 \\
\hline $0,005-0,1$ & 32849 & 2795 & 2711 & 84 & 32915 & 59 & 63 & -4 \\
\hline $0,1-0,2$ & 5467 & 504 & 485 & 19 & 5613 & 11 & 12 & -1 \\
\hline $0,2-0,5$ & 6308 & 632 & 555 & 77 & 6342 & 27 & 13 & 14 \\
\hline $0,5-1$ & 3202 & 336 & 263 & 73 & 3425 & 23 & 7 & 16 \\
\hline $1-2$ & 1608 & 215 & 4 & 84 & 1914 & 26 & 4 & 22 \\
\hline$>2$ & 679 & 83 & 131 & 39 & 905 & 30 & 2 & 28 \\
\hline Total & $\mathbf{8 6 5 7 2}$ & $\mathbf{7 5 7 8}$ & $\mathbf{7 2 0 3}$ & $\mathbf{3 3 5}$ & $\mathbf{8 6 5 7 2}$ & $\mathbf{2 4 9}$ & $\mathbf{1 6 6}$ & $\mathbf{8 3}$ \\
\hline
\end{tabular}

De même on constate un effet de l'age au moment de l'exposition : l'excès de risque relatif par $\mathrm{Sv}$ est plus élevé pour une exposition à un âge jeune.

Ces données mettent en évidence l'existence de facteurs modifiants la relation dose-effet (sexe, age à l'exposition, age atteint). La plupart de ces personnes ont reçu des doses faibles, mais à très fort débit de dose, ce qui est différent des conditions habituelles d'irradiation des travailleurs qui reçoivent de faibles doses à très faible débit tout au long de leur carrière professionnelle.

Les résultats de ces études sont compatibles avec une relation linéaire entre l'exposition aux radiations et l'excès de tumeurs solides à partir d'une exposition correspondant à environ $200 \mathrm{mSv}$. La relation doseeffet des personnes exposées à des faibles doses (de 5 à $200 \mathrm{mSv}$ ) ne met en évidence ni l'existence d'un seuil, ni une incompatibilité avec une relation quadratique. Pour les leucemies radioinduites, l'analyse de l'incidence des cancers chez les survivants des bombardements d'Hiroshima et de Nagasaki suggère que les doses infé- rieures à $150 \mathrm{mSv}$ ne conduisent pas à une augmentation du risque. Un tel seuil n'a pas été mis en évidence pour les cancers solides, mais il ne peut être exclu au dessous de $60 \mathrm{mSv}$.

La controverse scientifique est donc de savoir si la linéarité reste l'hypothèse la plus vraisemblable pour l'évaluation du risque pour des doses cumulées et des débits de dose inférieurs à $200 \mathrm{mSv}$.

Afin de prédire le risque associé à une exposition aux faibles doses, les épidémiologistes ont établi des relations dose-effet à partir d'études incluant des sujets qui ont reçu des doses variables, mais souvent bien supérieures à $100 \mathrm{mSv}$. Les recommandations internationales ont par conséquent été élaborées extrapolant pour les faibles doses ce qui était observé aux fortes doses et en utilisant principalement une relation dose-effet linéaire. Dans le domaine des faibles doses, la puissance des tests utilisés pour comparer des modèles de relation dose-effet alternatifs au modèle linéaire, est très faible. Ces doses n'ont que peu d'influence sur l'estimation de la forme de la relation dose-effet, qui 
est largement déterminée par les effets des doses nettement supérieures.

Le rapport conjoint des Académies de médecine et des sciences en France (Tubiana et al., 2005) estime qu'il n'existe aucune preuve épidémiologique d'un effet cancérogène chez l'enfant ou l'adulte pour des doses inférieures à $100 \mathrm{mSv}$. II y a débat pour ce qui concerne l'exposition du fœtus, le Comité Scientifique des Nations Unies pour l'Étude des Effets des Rayonnements lonisants (UNSCEAR) et le BEIR VII (2006) considèrent qu'un excès de leucémies et éventuellement des autres cancers de l'enfant est établi à partir d'exposition de 10 à $20 \mathrm{mSv}$ in utero, le Centre International de Recherche sur le Cancer (CIRC) et le rapport des Académies émettent des réserves sur la validité des conclusions et de toute manière considèrent que les résultats ne peuvent en être extrapolés à l'enfant.

\section{RÉPARATION}

Les lésions dues aux rayonnements ionisants sont les cassures simplebrin et double-brin de la double hélice d'ADN (Fig. 2). Les radiations induisent de manière aléatoire ces lésions de l'ADN.

Le nombre de lésions de l'ADN est proportionnel à la dose.

La plupart de ces lésions sont réversibles, mais celles qui résultent d'un rayonnement ionisant dense (proton ou particule alpha, par exemple) sont en général plus difficilement réparables que celles d'un rayonnement moins ionisant (rayons $X$ ou gamma, par exemple). Par conséquent, les rayonnements à forte densité d'ionisation (c'est-à-dire à TEL élevé) ont ordinairement une efficacité biologique relative (EBR) supérieure à celle des rayonnements à faible densité d'ionisation (à TLE peu élevé) pour la plupart des lésions. Les dommages non réversibles subis par l'ADN peuvent donner lieu à des mutations dont la fréquence est d'environ $10^{-5}$ à $10^{-6}$ par locus et par Gy.

Les effets des génotoxiques comme les rayonnements ionisants se traduisent en termes d'altérations irréversibles du génome (mutations) avec pour conséquence une augmentation des anomalies héréditaires et de la fréquence des cancers.

Comparées au vieillissement tissulaire, les radiations accélèrent l'accumulation des mutations. Après radiothérapie et irradiations à forte dose, on observe un apport brutal de mutations récessives. Le risque de seconde mutation pour induire un cancer est alors lié au vieillissement ultérieur.

Aux faibles doses ou faibles débits de dose, le système enzymatique est suffisant pour réparer les lésions avec peu de réparations fautives. Au débit de dose élevé, le processus de la réparation des bases est inductible, avec déclenchement d'un système qui répare mais augmente la proportion de réparations fautives.

Il apparaît de plus en plus vraisemblable que certaines formes d'inactivation cellulaire et d'aberrations chromosomiques radio-induites soient engendrées par une catégorie spécifique d'événements physiques primaires, dits événements critiques. Ces derniers seraient responsables de cassures double-brin d'ADN complexes, difficilement réparables par la cellule. 


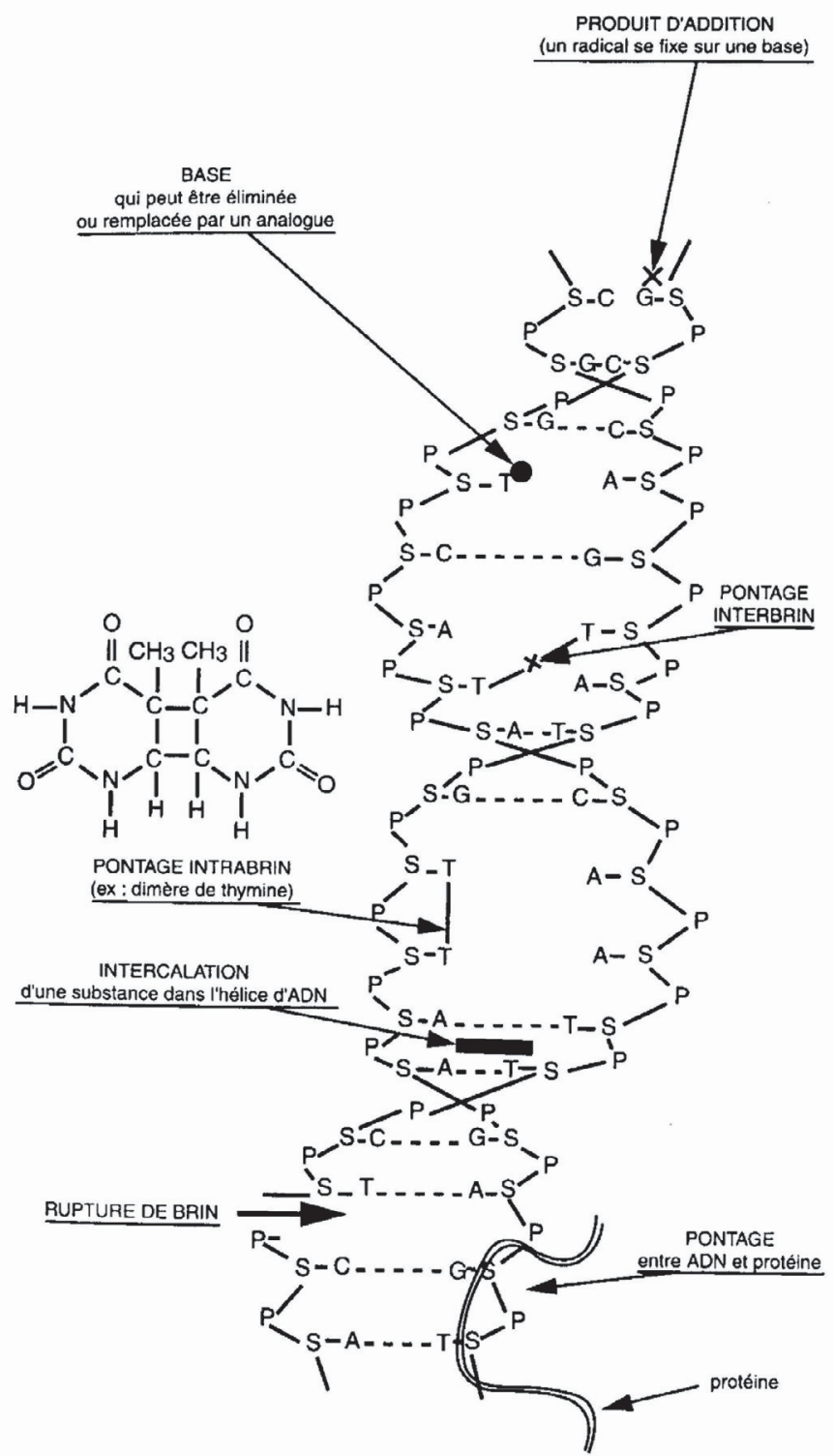

Fig. 2. Schéma simplifié de l'ADN avec certaines lésions types.

Fig. 2. Simplified diagram of DNA with typical damage. 
Tableau 2. Mutations rencontrées dans les tumeurs radioinduites.

Table 2. Mutations encountered in radiation induced tumors.

\begin{tabular}{|l|l|l|}
\hline Mutations & Type & Fréquence \\
\hline Non-sens : stop & récessive & fréquent \\
\hline Petite délétion & récessive & fréquent \\
\hline Gène de fusion & dominant & rare \\
\hline $\begin{array}{l}\text { Faux sens } \\
\text { activation }\end{array}$ & dominant & rare \\
\hline
\end{tabular}

Cette réparation fautive, à l'origine de mutations d'expression récessive (Tab. 2), à l'exception du cancer de la thyroïde chez l'enfant, aboutit le plus souvent à la perte fonctionnelle du gène concerné et renforce l'attention apportée aux gènes suppresseurs dans la conception générale de la cancérisation radio-induite.

L'homéostasie dans un tissu adulte normal est un effet caractérisé par un équilibre entre le nombre de cellules proliférantes, différenciées et apoptotiques. Tous les facteurs capables de modifier cet équilibre vers une prolifération cellulaire incontrôlée sont des cancérogènes comme les facteurs induisant des mutations dans les gènes contrôlant le cycle cellulaire, le rythme de prolifération ou l'induction d'apoptoses. Dans les cellules, différents systèmes de signalisation sont activés après la traversée d'un électron. Les recherches poursuivies depuis vingt ans sur la réparation de l'ADN ont montré que des systèmes de détection cheminent constamment le long des molécules d'ADN et alertent, en cas de lésion, des mécanismes de réparation. Les lésions de l'ADN ou les modifications de la conformation de la chromatine sont détectées par des protéines de signalisation dont l'acti- vité est modulée par le nombre de lésions en fonction de la dose, du débit de dose et du Transfert d'Énergie par unité de Longueur (TEL). Ces protéines activent des transmetteurs qui sont des phosphokinases, lesquelles modulent, à leur tour, l'action de protéines intervenant :

- soit dans le contrôle du cycle cellulaire (dont l'arrêt favorise la réparation),

- soit dans la réparation de l'ADN,

- soit dans le déclenchement de l'apoptose.

\section{INSTABILITÉ GÉNOMIQUE}

C. Streffer avait établi en 1984 que les mutations retrouvées tardivement après 20 à 30 divisions cellulaires dans les fibroblastes de l'embryon de souris irradiés, avant l'implantation dans l'utérus, ne correspondaient pas aux mutations initialement induites.

L'interprétation de ce phénomène correspondrait à une forme d'instabilité génétique, influencée par une prédisposition, et non liée directement à la production de doubles cassures de l'ADN provoquée par les rayonnements ionisants. Ce type d'instabilité a également été observé dans des cellules non exposées mais voisines (effet « bystander ») (Little, 2002). Un signal est émis par une cellule irradiée vers des cellules non irradiées permettant ainsi l'observation d'un effet biologique dans les cellules non exposées.

Depuis cette observation initiale, le mécanisme conduisant à l'instabilité du génome est devenu un point crucial en matière de cancérogenèse en général et de controverse scientifique sous-tendant le concept de linéarité 
entre la dose et l'effet en particulier. L'instabilité génomique résultant de multiples interactions, même à distance de la cellule irradiée, est l'équivalent d'une intoxication. La conséquence de l'acquisition de l'instabilité génomique est la production de mutations que l'irradiation ne produit pas initialement. La persistance de l'instabilité génétique au travers de multiples divisions cellulaires peut ainsi se transmettre par atteinte des cellules germinales à la descendance. Comme l'a souligné Roland Masse (Masse, 2000), « en matière de cancérogenèse, la notion d'instabilité génétique permet de réconcilier la valeur numérique de la probabilité combinée des multiples étapes (c'est-à-dire de mutations) nécessaires à transformer une cellule normale en cellule cancéreuse, avec celle connue de $n$ fois une mutation quelconque, spontanée ou induite par irradiation ".

Les hypothèses actuelles de cancérisation s'expliquent plus par un « effet de champ » et de la communication inter cellulaire (effet by-stander) que par l'irradiation d'un seul gène ou d'une seule cellule. À l'échelle cellulaire, la taille de la cible nécessaire pour acquérir l'instabilité semble incompatible avec la taille d'un gène. Un signal est émis par une cellule touchée vers des cellules non irradiées permettant ainsi l'observation d'un effet biologique dans les cellules non exposées.

À l'échelle du tissu, il faut souligner le rôle des contrôles par les cellules voisines (inhibition de la prolifération, échange de molécules de signalisation et de régulation par les canaux de jonctions intercellulaires, effet « bystander », sécrétion par les cellules voi- sines et le stroma de facteurs de régulation). Ces interactions entre cellules sont permanentes et jouent un rôle crucial dans la construction des tissus pendant l'embryogenèse, la croissance, le renouvellement de certains tissus à l'âge adulte et la réparation des tissus lésés. Dans le processus de cancérogenèse, il existe des interactions multiples entre la cellule siège d'un événement génétique potentiellement oncogène, les cellules voisines de même nature, et les divers éléments qui constituent le microenvironnement en particulier les cytokines qui peuvent, selon le contexte, ralentir ou accélérer cette transformation. Le microenvironnement peut arrêter ou stimuler la prolifération des clones de cellules en voie de cancérisation et agir sur l'instabilité génétique.

Les effets by-stander ont été observés à bas ou haut TEL . Les principales conséquences observées ont été soit :

- des mutations de gènes spécifiques (Nagasawa et Little, 1999) et $90 \%$ des mutations sont ponctuelles (à l'inverse des délétions observés dans les cellules irradiées) (Huo et al., 2001) ;

- des formations de micro noyaux dû aux dommages de l'ADN responsable de l'apoptose dans les cellules by-stander (Prise et al., 1998, Belyakov et al., 2001);

- des modulations de l'expression de certains gènes où p53 joue certainement un rôle central, mais aussi CDC2, CyclineB1 et rad51 (Azzam et al., 1998) ;

- mais également l'augmentation de la transformation maligne (Sawant et al., 2001) et de «cell killing ». 
Nagasawa et Little (1999) ont montré que la relation dose-réponse par des fluences faibles de particules $\alpha$ était curvilinéaire pour l'induction de mutations au locus HPRT chez des cellules $\mathrm{CHO}$. La réponse curvilinéaire aux faibles doses (0-2 cGy) est le résultat de mutations survenant chez les cellules by-stander non irradiées.

À l'échelle cellulaire, la taille de la cible nécessaire pour acquérir l'instabilité semble incompatible avec la taille d'un gène. Elle ne se produirait qu'audelà d'un seuil pour créer le niveau cytotoxique nécessaire à l'accumulation des dommages du stress oxydant et des cassures double-brin; une instabilité peut être induite par irradiation de cellules par une seule particule alpha (microbeam) (Kadhim et al., 2001) et peut être saturée à plus haute dose.

\section{RADIOSENSIBILITÉ INDIVIDUELLE}

Vers la fin des années 80 , grâce au développement de la génétique humaine, en particulier à l'utilisation des marqueurs polymorphes, la dimension du caractère héréditaire du cancer a pu être prise en compte et a conduit à l'identification de gènes de prédisposition à plusieurs cancers fréquents tels le sein, le colon, la peau. Aujourd'hui, on réalise que toute mutation transmissible de gènes impliqués dans la réparation de l'ADN, dans la prolifération et le cycle cellulaire (p53 par exemple) peut entraîner une sensibilité accrue aux effets carcinogènes et déterministes des radiations.

La découverte des gènes suppresseurs de tumeurs et de leur fonction a amené à identifier les sujets prédisposés aux cancers car porteurs constitutionnellement d'un allèle muté de l'un de ces gènes. C'est la mutation somatique du second allèle, avec le vieillissement ou sous l'effet des radiations même aux faibles doses, qui peut entraîner la cancérisation à partir de la cellule double mutante.

Les variations de radiosensibilité individuelle proviennent très probablement de la variabilité interindividuelle à réparer les lésions de l'ADN ou à éliminer les cellules endommagées.

Le nombre et le type de gènes impliqués sont probablement très importants. Ce nombre et ces variations pourraient expliquer la plus ou moins grande sévérité de l'hypersensibilité aux radiations ionisantes. La susceptibilité individuelle aux effets stochastiques et déterministes des rayonnements ionisants existe et ces sujets représentent une minorité de la population générale. II apparaît qu'une composante importante de la sensibilité des individus peut être liée à des facteurs génétiques constitutifs. Deux maladies sont particulièrement bien connues : I'Ataxia Télangiectasia (AT) et la maladie de Fanconi. Ces maladies autosomiques récessives sont heureusement très rares, voir rarissimes $(A T=$ $1 / 100000$ ). L'ataxie telangectasie (AT) fait partie des syndromes caractérisés par une hypersensibilité aux radiations, pour lesquels un facteur génétique a été caractérisé. D'autres conditions de radiosensibilité extrême ont également été décrites, sans qu'une relation directe avec une mutation de prédisposition génétique ou acquise n'ait été identifiée.

Outre les anomalies génétiques constitutives (prédisposition) ou 
acquises par l'irradiation, une composante importante de la variabilité de la radiosensibilité individuelle est liée à des facteurs épigénétiques.

Les conclusions du Comité 1 de la CIPR (Publication 79 de la CIPR) sont «le risque individuel sur la population génétiquement prédisposée n'est que faiblement augmenté par de faibles doses même si la radiosensibilité est 100 fois plus élevée ». Du fait de leur faible prévalence dans la population, la prise en compte de ces cas ne change pas l'estimation du risque pour la population générale.

Pour le risque individuel, il n'existe pas de réglementation quant à l'utilisation de test de dépistage en milieu professionnel notamment à l'embauche. L'une des raisons avancées est le fait qu'il ne faut pas reporter sur les «gènes des salariés " la responsabilité et les obligations de protection face aux rayonnements ionisants qui incombent à l'employeur.

La Loi $n^{\circ}$ 2002-303 du 4 mars 2002 Titre II article 4 relative aux droits des malades et à la qualité du système de santé stipule : "Nul ne peut faire l'objet de discriminations en raison de ses caractéristiques génétiques ». Par contre, s'il n'a pas été démontré aujourd'hui un risque supplémentaire en médecine diagnostique ou sur le plan professionnel en raison des faibles doses d'irradiation, il paraît évident que ces personnes en thérapie exposées à de fortes doses peuvent bénéficier d'un dépistage avant toute radiothérapie pour optimiser leur traitement et prendre en compte le risque de cancer secondaire non négligeable.

\section{LES EFFETS HÉRÉDITAIRES}

Les effets héréditaires résultent des effets de l'irradiation avant la conception (lésions des chromosomes de la lignée germinale (spermatozoïdes et ovules)). Ces lésions peuvent provoquer des anomalies dans la descendance de l'individu exposé.

Quoique bien documentés pour d'autres organismes vivants, les effets héréditaires de l'irradiation n'ont jamais été observés chez les humains. Les données humaines ont porté sur la descendance d'individus irradiés soit médicalement, soit accidentellement pendant une courte durée ou à la suite des deux bombardements nucléaires. Par ailleurs les études qui ont porté sur des populations qui vivent dans des zones à « forte » radioactivité ambiante sont contradictoires, et donc peu fiables, car il n'existe ni registre, ni suivi médical approprié dans les zones à forte radioactivité naturelle étudiées.

En conséquence, les données d'Hiroshima et de Nagasaki constituent donc la seule base (70000 grossesses entre 1948 et 1953) sur laquelle s'appuyer pour établir un seuil au-dessous duquel aucun effet héréditaire n'est observé. Mais la validité des études sur les descendants des irradiés d'Hiroshima et de Nagasaki peut être remise en cause si l'on considère que les mutations induites étaient, en grande majorité, récessives (Dutrillaux, 1998).

Aucun effet ne serait attendu en première génération (génération de 1946 à 1966). On n'observerait que des hétérozygotes, porteurs de mutations variées, différentes les unes des autres. Chacun d'entre eux serait en mesure de transmettre, une fois sur deux, sa mutation. C'est uniquement 
à la cinquième génération (2026-2046) qu'une conséquence perceptible pourrait être attendue. Cette hypothèse expliquerait l'absence d'augmentation de pathologie transmissible sur la cohorte de Hiroshima et Nagazaki.

Les altérations de l'ADN induites et les conséquences comme les lésions non transcrites, qui se traduisent par un simple polymorphisme de l'ADN, sont intéressantes à étudier.

Les minisatellites ont été testés comme marqueurs biologiques des irradiations à faible dose. Une étude publiée en 1996 (Dubrova et al., 1996) suggérait que les populations ayant subi les conséquences de l'accident de Tchernobyl (population chroniquement exposée) ont un taux de mutation accru d'un facteur 2 aux locus chromosomiques des minisatellites dits hypermutables. Récemment, le taux de mutation germinale naturel des minisatellites hypermutables a montré être identique dans deux populations différentes (ukrainienne et britannique) (Livshits et al., 2001). Ceci va à l'encontre de l'existence de variations du taux moyen de mutation liées à des différences d'origine ethnique et à des différences de mode de vie, et renforce les conclusions de l'étude déjà publiée.

Un deuxième point a été étudié, le problème des pères exposés à Tchernobyl (liquidateurs) aux rayonnements ionisants et la transmission de mutations génétiques à leur descendance. Le taux de transmission de mutations d'allèles mutants aux enfants dont le père a été exposé est le même que celui de la population témoin. Cependant, lorsque l'analyse distingue les enfants conçus pendant la période d'exposition ou plus tard, le taux de mutation dans le premier groupe apparaît 1,5 fois plus élevé que dans le second. Cette différence n'est pas statistiquement significative (car l'un des problèmes de ce type d'étude rétrospective est l'hétérogénéité des doses reçues par le groupe d'homme étudié).

Cependant, cette observation, qui actuellement est l'objet de discussions, est en accord avec les expériences parallèles réalisées chez la souris. Dans ce modèle, la mesure du taux de mutation de minisatellites après irradiation montre que celui-ci est accru par l'irradiation, plus particulièrement au stade méiotique de la maturation de la lignée germinale, et que cet accroissement est également transitoire. Mais l'extrapolation vers l'homme des résultats obtenus chez la souris doit être considérée avec prudence en raison de la différence de comportement des minisatellites chez l'homme et chez d'autres mammifères.

\section{Estimation du risque}

Par conséquent, en l'absence d'effet observé chez l'homme, l'estimation des risques d'effets héréditaires des rayonnements dépend dans une grande mesure de l'extrapolation des conclusions tirées de l'observation de souris et d'autres animaux de laboratoire. Dans son rapport de 2001, I'UNSCEAR estime sur la base également d'hypothèses de prudence que, pour une irradiation des parents de 100 mGy, on attendrait un excès d'environ 400 maladies à composante héréditaire par million d'habitants, soit une incidence de $0,4 \%$ à la première génération et $0,2 \%$ à la génération suivante. Ceci représenterait moins d'un 
dixième du risque de cancer mortel pour la même irradiation.

Le risque pour la descendance a été estimé par prudence par la CIPR dès 1977 (Publication 26) à $25 \%$ pour un sievert; ce risque a été depuis diminué en l'absence d'arguments nouveaux, à $20 \%$ dans la Publication 60 de la CIPR et serait de $5 \%\left(0,5 \times 10^{-2} \mathrm{~Sv}^{-1}\right)$ dans la future publication et ceci toujours par précaution.

\section{AUTRES PATHOLOGIES}

Les maladies radio-induites autres que les cancers affectant les systèmes cardiovasculaire, respiratoire et digestif étaient jusqu'alors considérées comme appartenant à la famille des effets déterministes, avec des seuils de dose relativement élevés estimés entre 4 à 5 Gy (suivant l'affection et l'organe), il apparaît maintenant que ces maladies peuvent être induites par des doses inférieures. Ce point est particulièrement mis en évidence par le suivi à long terme des survivants d'Hiroshima et Nagasaki (Life Span Study) et de nouvelles études sur des populations exposées à des doses relativement faibles sont en cours.

\section{CONCLUSION}

Actuellement, bien que commode pour la gestion des risques, I'hypothèse de linéarité de la relation doseeffet n'est pas vérifiée pour tous les modèles. En particulier, dans le domaine des faibles débits de doses délivrées par des émetteurs de faible TEL, cette hypothèse est très controversée à la lumière des observations récentes, notamment celles concernant les mécanismes conduisant à l'instabilité génétique, la radiosensibilité individuelle et à l'inductibilité de la réparation de I'ADN. Le problème des émetteurs à forts TEL reste posé.

$\mathrm{Au}$ vue des connaissances actuelles, on pourrait subdiviser tel que le propose le rapport conjoint de l'Académie de médecine et des sciences (Tudiana et al., 2005) les effets des faibles doses sur la cellule selon la réponse cellulaire et tissullaire :

Quand la dose ou le débit de dose sont très faibles, les systèmes de détection et de signalisation intracellulaire ne sont pas déclenchés et les cellules s'abstiennent de toute réparation des cassures doubles brins (CDB), ce qui entraîne la mort de la plupart de ces cellules au moment de la mitose. Cette élimination protège l'organisme contre les cellules potentiellement susceptibles de devenir cancéreuses.

Pour des doses un peu plus élevées (quelques $\mathrm{mSv}$ et environ $10 \mathrm{mSv}$ ), la cellule réagit en stimulant des mécanismes de défense (capteurs de radicaux et antioxydants, déméthylase), des processus de réparation et en déclenchant éventuellement la mort cellulaire programmée (apoptose) de cellules potentiellement mutantes dont l'ADN a été endommagé. Ce phénomène apparaît pour des doses de l'ordre de l'irradiation naturelle, ce qui permet d'en réduire ou d'en supprimer les effets potentiellement nocifs. II peut avoir pour origine soit la cellule lésée, soit les cellules saines de son environnement.

Pour des doses et débits de doses plus élevés (au-delà de quelques dizaines de $\mathrm{mSv}$ ), l'intégrité 
fonctionnelle du tissu ne permettrait pas d'éliminer les cellules endommagées. Les mutations peuvent s'accumuler à travers le processus d'instabilité génomique. En revanche, le nombre de cellules lésées ne permet plus leur élimination pure et simple. Les mutations peuvent s'accumuler à travers le processus d'instabilité génomique. Les systèmes de réparation sont alors activés pour permettre la survie cellulaire et préserver les fonctions des tissus ce qui aboutit généralement à une réparation fidèle mais expose les cellules à un risque de réparation fautive. Le risque de réparation fautive croît avec la dose et le débit de dose en fonction du nombre de lésions de l'ADN simultanément présentes dans la cellule.

À partir de quelques centaines de mGy, une prolifération cellulaire compensatrice est stimulée, ce qui augmente encore le risque de réparation fautive et permet à certaines cellules d'échapper aux processus de maintien de l'intégrité tissulaire et au contrôle de la prolifération.

Ces mécanismes d'échappement varient considérablement selon les tissus, la nature des cellules initiées (cellules souches ou progéniteurs) et le type de tumeur. L'inhibition de la division cellulaire se manifeste rapidement après l'exposition et varie donc tant avec le niveau de dose qu'avec le débit de dose reçu. Mesurée en fonction de la capacité de prolifération, la survie des cellules en cours de division tend à décroître de façon exponentielle avec l'augmentation de la dose, 1 à 2 Gy suffisant en général pour réduire de moitié le nombre de cellules survivantes.
La probabilité de mutation par unité de dose n'est donc pas constante quels que soient la dose et le débit de dose, et le processus de cancérogenèse, une fois initié dans une cellule, n'évolue pas indépendamment des lésions éventuelles des cellules environnantes. Les relations entre la cellule lésée et les cellules environnantes jouent un rôle essentiel. Des systèmes de signalisation intercellulaire informent chaque cellule sur le nombre de cellules environnantes ayant été lésées. Le processus de cancérogenèse se heurte alors à des mécanismes efficaces de défense à l'échelle du tissu. Les mécanismes qui agissent dans l'embryogenèse et pour diriger la réparation tissulaire après une agression semblent intervenir pour contrôler la prolifération d'une cellule, même quand celle-ci est devenue précancéreuse et autonome.

Enfin, les systèmes de surveillance mis en œuvre par les cellules saines de l'organisme sont capables d'éliminer des clones de cellules transformées, comme le montrent les échecs des greffes de cellules tumorales ainsi que la forte augmentation de la fréquence de certains cancers chez les sujets immunodéprimés.

Des méthodes d'analyses globales de la réponse cellulaire après irradiation existent comme la méthode appelé transcriptome permettant d'étudier la transcription des ARN dans une cellule. Elles permettent de donner une image de la cellule à un instant donné de l'expression des gènes dans des cellules. Elle a permis de montrer que les faibles doses d'irradiation entraînaient des modifications d'expression et que certains des gènes 
induits ou réprimés n'étaient pas systématiquement induits ou réprimés aux doses plus fortes. Ainsi, ces techniques d'analyse globale ouvrent de nouvelles perspectives pour analyser de manière objective les effets des faibles doses et donc d'établir des bases rationnelles pour étudier les risques aux faibles doses et en conséquence les normes de radioprotection. Jusqu'à présent, ces normes, qui ont pour objectif de limiter les effets biologiques des radiations, étaient basées sur les données extrapolées à partir des résultats acquis aux fortes doses, considérant a priori que les mécanismes impliqués étaient identiques.

Ces faits scientifiques pourraient, si les législateurs en tiennent compte, avoir une conséquence sur l'évolution future des limites d'exposition réglementaires.

\section{RÉFÉRENCES BIBLIOGRAPHIQUES}

BEIR VII 2006 Phase 2 Board on Radiation Effects Research (BRER); Health Risks from Exposure to Low Levels of Ionizing Radiation: http://newton.nap.edu/books/ 030909156X/html/

Belyakov O.V., Malcolmson A.M., Folkard M., Prise K.M., Michel B.D. 2001 Direct evidence for a bystander effect of ionizing radiation in primary human fibroblasts. Br. J. Cancer 84, 674-679.

CIPR 1977 CIPR26, Recommandations of the international commission on Radiological Protection, Pergamon, Annals of the ICRP, Vol. 1 (3).

CIPR 1990 CIPR60, Recommandations of the international commission on Radiological Protection, Pergamon, Annals of the ICRP, Vol. 12 (1-3).

CIPR 1998 CIPR79, Genetic susceptibility to cancer, Pergamon, Annals of the ICRP CIPR. Vol. 28 (1-2).
Dubrova Y.E., Nesterov V.N., Krouchinsky N.G., Ostapenko V.A., Neumann R., Neil D.L. \& Jeffreys A.J. 1996. Human minisatellite mutation rate after the Chernobyl accident. Nature. Apr 25, 380(6576):683-6.

Dutrillaux B. 1998. Peut-on savoir si un cancer est dû à la radioactivité ?, La Recherche, Vol. 308, 68-77.

Huo L., Nagasawa H., Little J.B. 2001 HPRT mutants induced in bystander cells by very low fluences of alpha particles result primarily from point mutations. Radiation Reasearch. 156, 521525.

Kadhim M.A., Marsden S.J., Goodhead D.T., Malcomson A.M., Folkard M., Prise K.M., Michael B.D. Long-term genomic instability in human lymphocytes induced by single-particle irradiation. Radiation Research 2001. 155, 122126.

Little J.B. 2002. Instabilité génomique et effet "bystander" induit par les rayonnements ionisants : implications pour la radioprotection. Radioprotection, Vol. 37 (3), 261-277.

Livshits L.A., Malyarchuk S.G., Kravchenko S.A., Matsuka G.H., Lukyanova E.M., Antipkin Y.G., Arabskaya L.P., Petit E., Giraudeau F., Gourmelon P., Vergnaud G. \& Le Guen B. 2001. Children of Chernobyl Cleanup Workers do not Show Elevated Rates of Mutations in Minisatellite Alleles. Radiat Res. 155(1): 74-80.

Masse R. 2000 Rayonnements ionisants. Comptes rendus de l'Académie des Sciences. Série III. Sciences de la vie, 323, 7, 633-640, 8 p.

Michel L.A., Donckier J.E. 2002 Thyroid cancer 15 years after Chernobyl. Lancet. Jun. 1, 359(9321):1947.

Nagasawa H., Little J.B. 1999 Unexpected sensitivity to the induction of mutations by very low doses of alpha-particle radiation : Evidence for a bystander effect. Radiation research, 152, 552-557.

NCRP 2001 Evaluation of the linearnonthreshold dose-response model for ionizing radiation NCRP report 
136. National Council on Radiation Protection and Measurements, $287 \mathrm{p}$.

Preston D.L., Shimizu Y., Pierce D.A., Suyama A. Mabuchi K. 2003 Studies of mortality of atomic bomb survivors. Report 13: Solid cancer and noncancer disease mortality: 1950-1997. Radiat Res. Oct. 160 (4): 381-407.

Prise K.M., Belyakov O.V., Folkard M., Michael B.D. 1998 Studies of bystander effects in human fibroblasts using a charged particle microbeam. Int. J. Radiat. Biol. 74, 793-798.

Sawant S.G., Randers-Perhrson G., Geard C.R., Brenner D.J., Hall E.J. 2001 The bystander effect in radiation oncogenesis : I. Transformation in $\mathrm{C} 3 \mathrm{H} 10 \mathrm{~T} 1 / 2$ cells in vitro can be initiated in the unirradiated neighbors of irradiated cells. Radiation Reasearch. 155, 397-401.

Tubiana M., Aurengo A., Averbeck D., Bonnin A., Le Guen B., Masse R., et al.
2005 La relation dose-effet et l'estimation des effets cancérogènes des faibles doses de rayonnements ionisants, rapport commun Académie des Sciences et Académie Nationale de Médecine. Éditions Nucléon diff. EDP Sciences ; http://www.academiemedecine.fr/upload/base/rapports_228 fichier lie.rtf

http://www.academie-sciences.fr/ publications/rapports/pdf/ dose_effet_07_04_05.pdf

UNSCEAR 2000 Sources and effects of ionizing radiation. UNSCEAR 2000 report to the General Assembly, with scientific annexes. Vol. I. Sources. Nations Unies, 1, $654 \mathrm{p}$.

UNSCEAR 2000 Sources and effects of ionizing radiation. UNSCEAR 2000 report to the General Assembly, with scientific annexes. Vol. II. Effects. Nations Unies, 2, $566 \mathrm{p}$. 